\title{
Acoustofluidic Separation of Proteins using Aptamer-Functionalized Microparticles
}

\author{
Muhammad Afzal, ${ }^{1, \$}$ Jinsoo Park, ${ }^{2, \$}$ Jessie S. Jeon, ${ }^{1}$ Muhammad Akmal, ${ }^{3}$ Tae-Sung Yoon, ${ }^{4}$ and Hyung \\ Jin Sung, ${ }^{1 *}$ \\ ${ }^{1}$ Department of Mechanical Engineering, KAIST, 291 Daehak-ro, Yuseong-gu, Daejeon 34141, Korea \\ ${ }^{2}$ School of Mechanical Engineering, Chonnam National University, 77 Yongbong-ro, Buk-gu, Gwangju 61186, Korea \\ ${ }^{3}$ Department of Materials Engineering, KAIST, 291 Daehak-ro, Yuseong-gu, Daejeon 34141, Korea \\ ${ }^{4}$ Department of Proteome Structural Biology, KRIBB, 125 Gwahak-ro, Yuseong-gu, Daejeon 34141, Korea \\ ${ }^{\$}$ These authors contributed equally in this work. \\ *To whom correspondence should be addressed. E-mail: hjsung@kaist.ac.kr
}

\section{Hemocytometer Quantification}

To demonstrate the quantitative experimental analysis for acoustofluidic tri-separation of proteins, the efficiency of separated samples was measured in terms of their purity and recovery rate using a hemocytometer particle quantification technique. In this regard, three sets of independent experiments for acoustofluidic protein separation were conducted again and the separation efficiency of the collected samples was measured by calculating both separation purity and recovery rate in five repeated hemocytometer measurements for each sample (Figure S1). The purity and recovery rate were measured for before and after separation experiments. In our experiments, the total number of 1,744,000/ml particles were measured in initial sample injected into the sample inlet with a composition of 976,000/ml (55.96\%) thrombin-conjugated green particles and 768,000/ml (44.04\%) IgE-conjugated orange particles. After the separation, the number of particles were calculated in all three samples collected at outlets. In sample at outlet 3 , the green particles were $954,723 / \mathrm{ml}(97.82 \%)$ while orange particles were 9,216/ml (1.20\%). In sample collected at outlet 2 , the number of orange particles were $739,661 / \mathrm{ml}(96.31 \%)$ while the green particles were $8,589 / \mathrm{ml}(0.88 \%)$. In outlet 1 sample, the green particles were 12,688/ml (1.30\%) while the number of orange particles were $19,123 / \mathrm{ml}(2.49 \%)$. With the help of these numerical values of particle calculations at all three outlets after separation, the purity of thrombin-conjugated green particles was calculated to be $99.04 \%$ and of IgE-conjugated orange particles was $98.85 \%$. The recovery rate after separation was calculated to be $97.82 \%$ for thrombin-conjugated green particles and $96.31 \%$ for IgE- 
conjugated orange particles. These quantification measurements supported high separation efficiency of our proposed acoustofluidic protein tri-separation technique.

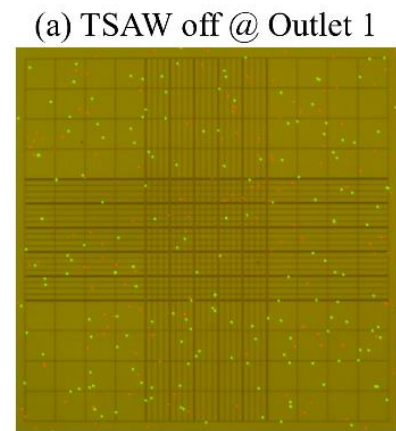

(d) TSAW on @ Outlet 1

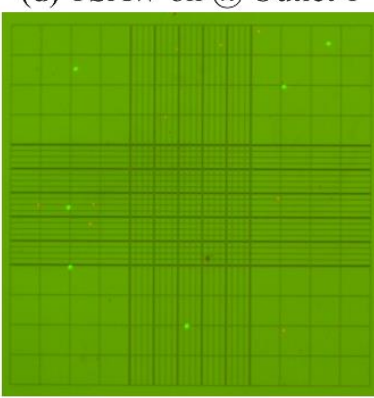

(b) TSAWoff@ Outlet 2

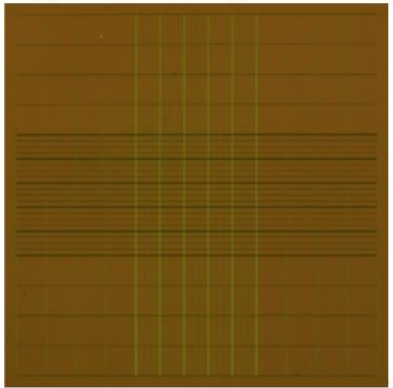

(e) TSAW on@ Outlet 2

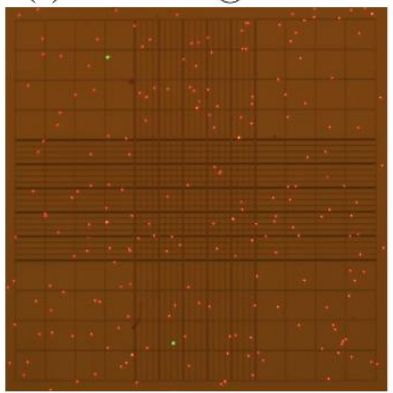

(c) TSAW off @ Outlet 3

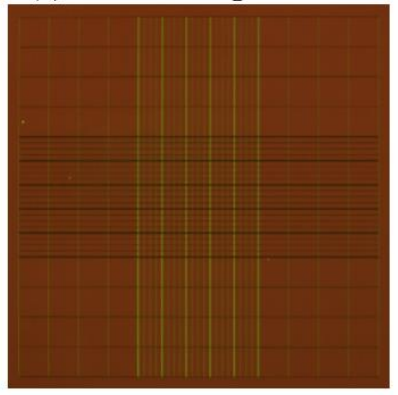

(f) TSAW on@, Outlet 3

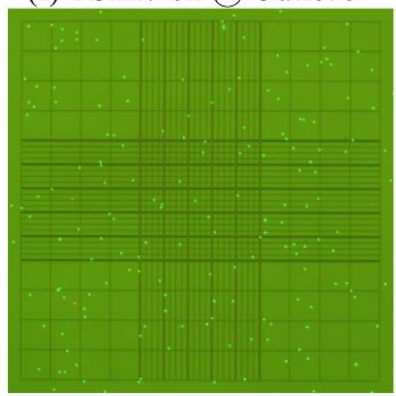

Figure S1. Hemocytometer images of the samples collected at the outlets. 